\title{
Despite noise, fractons remain unmoved
}

\author{
Localization in fractonic random circuits \\ Authors: Shriya Pai, Michael Pretko, and Rahul M. Nandkishore \\ arXiv:1807.09776
}

\section{Recommended with a Commentary by Daniel Arovas, University of California, San Diego}

In recent years, the subject of many-body quantum dynamics has attracted considerable interest among theorists. The goal largely has been to develop analytically or numerically tractable models from which one can deduce certain robust features associated with thermalization, quantum chaos, and 'scrambling' dynamics of quantum information (e.g., entanglement growth). One might think the first step in constructing such a model is to decide upon a Hamiltonian, but some essential aspects of unitary quantum evolution may be gleaned by examining the behavior of random quantum circuits (RQCs) [1], in which there is no Hamiltonian at all! Rather, the unitary evolution of a system is defined by a set of random $\mathcal{S}$-matrices (see Fig. 1), each of which evolves a fixed number $k$ of incoming qudit amplitudes into $k$ outgoing amplitudes, each qudit being a $q$-state entity (i.e. a generalized qubit, with $q=2 S+1$ for a spin- $S$ object). Each $\mathcal{S}$-matrix is thus a $q^{k} \times q^{k}$ unitary matrix, typically distributed according to the Haar measure. In such circuits, there are no locally conserved quantities. Yet the dynamics of quantum information, as adduced from the spreading of operators in time, or the behavior of 'out-of-time-ordered correlation functions' (OTOCs), is quite simple, propagating as a diffusively broadening front such that the characteristic spatial extent of any nontrivial local operator expands linearly with the 'butterfly velocity' $v_{\mathrm{B}}{ }^{*}$. Away from the front, relaxation is exponential. Such systems are ergodic - at long times, operator correlations approach their infinite temperature values. While these RQCs are purely noise-driven, their hydrodynamic description is believed to have applicability to deterministic (nonrandom) ergodic quantum systems, whose internal dynamics ostensibly generate endogenous noise.

When the random circuit is constructed so as to preserve a $\mathrm{U}(1)$ symmetry (charge conservation) [2], the exponential relaxation becomes diffusive for operators which have 'overlap' with the local charge density, and the tail of the propagating front is modified. Still, the system is ergodic, and is described by a Gibbs ensemble at late times.

Coincident with this work in many-body quantum dynamics, an exciting new class of quantum states has been discovered and studied: fracton phases [3]. While global charge conservation still permits local charge motion, in fracton systems the movement of elementary excitations is severely restricted; they are fully mobile only when agglomerated into

\footnotetext{
*Entanglement spreading is characterized by a separate 'entanglement velocity' $v_{\mathrm{E}}<v_{\mathrm{B}}$.
} 

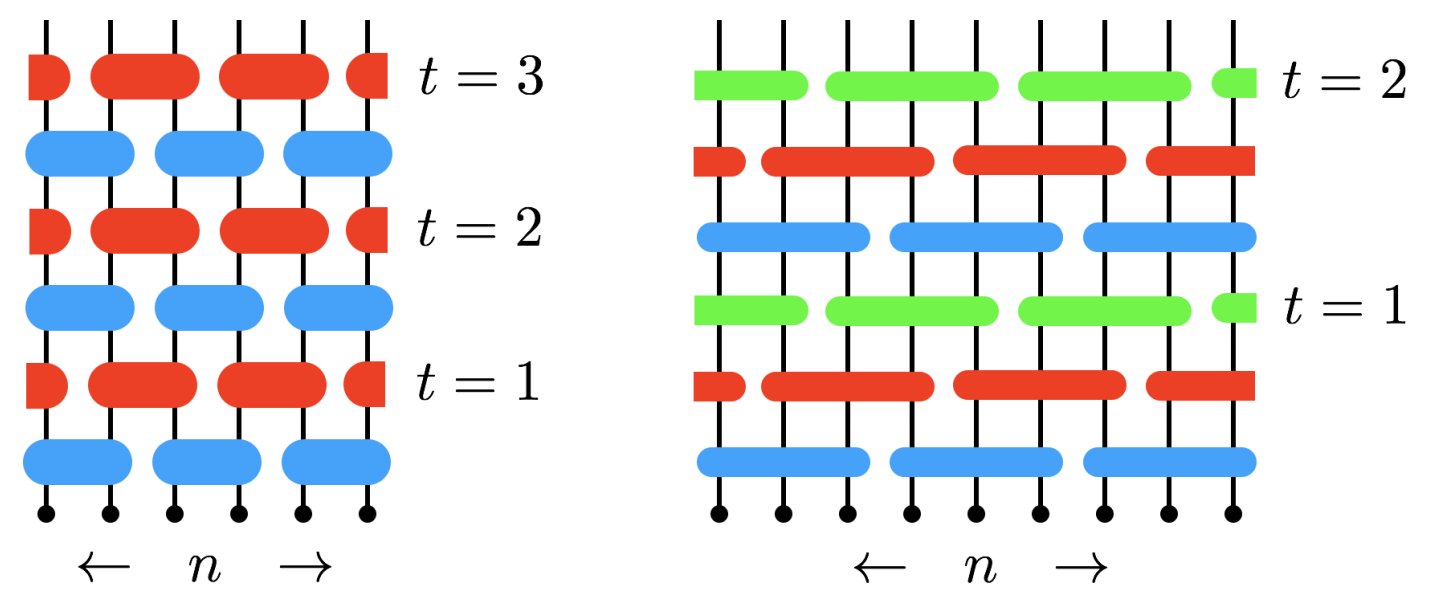

Figure 1: One-dimensional quantum circuits build from $k$-qudit gates, with $k=2$ (left) and $k=3$ (right). Each qudit is a $q$-state object. Spatial (horizontal, periodic) and temporal (vertical) coordinates are discrete.

multiparticle composites ${ }^{\dagger}$. Nevertheless, when placed in contact with a heat bath, fracton models thus far studied, while exhibiting strongly suppressed low-temperature charge mobility, nevertheless do thermalize, albeit on a time scale which is exponential or even super-exponential in $1 / T$ [4].

Pai, Pretko, and Nandkishore (PPN) construct and explore models of random unitary dynamics of one-dimensional fracton systems. Their basic idea is to constrain the dynamics in such a way that not only is $\mathrm{U}(1)$ charge $Q$ conserved, but dipole moment $P$ is as well. Individual charges cannot move independently, since their motion would change $P$. But, similar to the state of affairs in higher-dimensional fracton models (such as the $X$-cube model or Haah's code), assemblies of local charges - dipoles in this case - are mobile. The motivation is to construct noisy unitary quantum circuits with nonergodic phases, similar, for example, to that of many-body localization (MBL). Their simplest fractonic circuit resembles the right panel of Fig. 1, where each qudit is a $S=1(q=3)$ entity. The local charge on site $n$ is measured by the operator $S_{n}^{z}$. PPN's $\mathcal{S}$-matrices are three-qudit gates (of rank $q^{k}=3^{3}=27$ ), and are constructed such that $Q=\sum_{n} S_{n}^{z}$ and $P=\sum_{n} n S_{n}^{z}$ are preserved. Consequently, rather than being fully Haar-random, all their unitaries are almost diagonal in the $S^{z}$ basis, consisting of 19 random phases and four Haar-random $2 \times 2$ blocks. The latter, for each spatially consecutive triple of qudits, allow for the transitions $|+-0\rangle \leftrightarrow|0+-\rangle$ and $|-+0\rangle \leftrightarrow|0-+\rangle$, corresponding to individual dipole motion, as well as $|0+0\rangle \leftrightarrow|+-+\rangle$ and $|0-0\rangle \leftrightarrow|-+-\rangle$, corresponding to motion of fracton charge accompanied by the emission or absorption of a dipole.

The results are striking. While energy-conserving translationally-invariant fracton systems equilibrate, albeit slowly, PPN's random fractonic circuits are nonergodic, retaining memory of their initial conditions even at long times. Even though the dynamics allow for the motion of charges, and there is no energy conservation, fractonic charge in RQCs fails

${ }^{\dagger}$ As with topologically-ordered phases, gapped fracton phases exhibit degenerate ground states which are indistinguishable by local operator measurements. Their ground state degeneracies typically grow with system size (subextensively), while that for topologically ordered phases is of order $L^{0}$. 
to spread completely - a feature PPN attribute to the return statistics of low-dimensional random walks, similar to the phenomenon of weak localization, but insensitive to dephasing. It would thus be interesting to explore three-dimensional generalizations of PPN's models.

Numerical simulations of operator spreading are shown in Fig. 2, which shows the evolution of the 'right weight profile' $\rho_{\mathrm{R}}(n, t)=\sum_{\operatorname{right}(\Gamma)=n}\left|a_{\Gamma}(t)\right|^{2}$, where $\{\Gamma\}$ is a basis of operator strings, each of which is a product of local operators at each site. The time dependence of any operator $O$ can be expressed as $O(t)=\sum_{\Gamma} a_{\Gamma}(t) \Gamma$, and in the evaluation of right weights, only strings $\Gamma$ whose rightmost non-identity operator is at site $n$ contribute to the sum. The left panel shows the spreading of a single local dipole operator; the peak at the initial dipole position dissipates and emits a propagating and spreading front, similar to what is found in generic charge-conserving RQCs [2]. The right panel shows the spreading of a local charge operator. In this case, $\rho_{\mathrm{R}}(n, t)$ exhibits a lingering peak at the initial location of the operator. A traveling and spreading front is again emitted, but its tail exhibits a different power law behavior than for the dipole operator. In both cases, the right weight profile appears to be self-averaging with respect to the randomness.
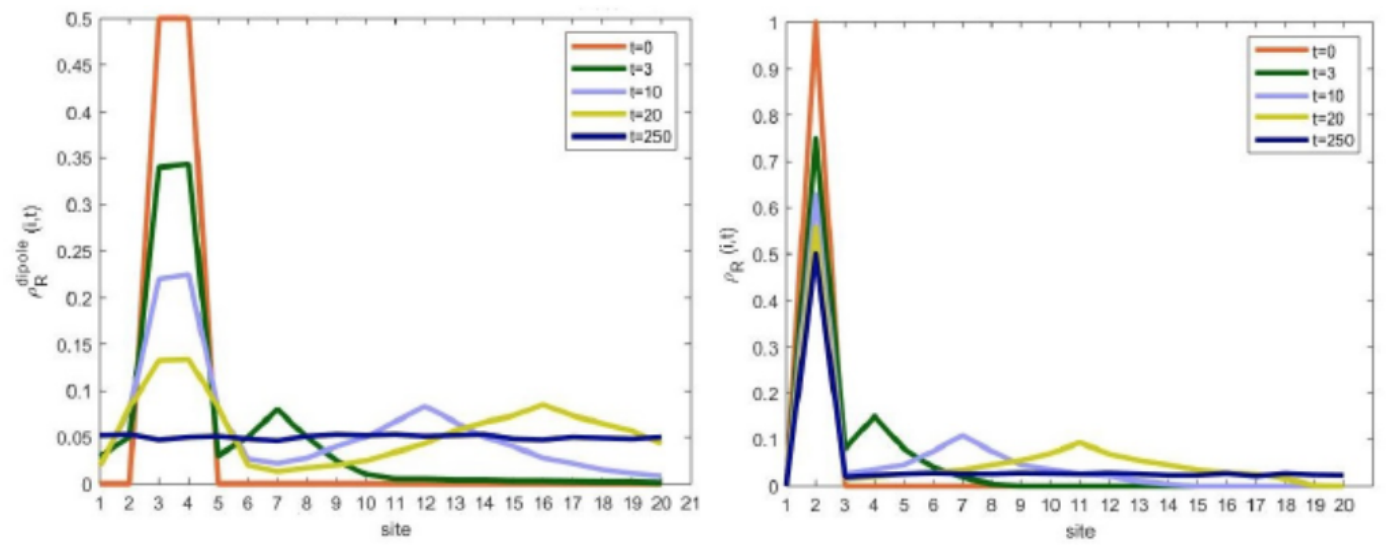

Figure 2: Local dipole (left) and local charge (right) operator spreading in random unitary fractonic circuits, as measured by the 'right weight profile' $\rho_{\mathrm{R}}(n, t)$.

Another key distinction in the fractonic circuits is their saturation entanglement entropy. Starting with an initial product state, the entanglement entropy $S_{\mathrm{E}}(t)$ across a cut typically rises linearly but eventually saturates. For generic RQCs exhibiting thermalization, the saturation value of $S_{\mathrm{E}}(t)$ is close to maximal, scaling linearly with the partition size. By contrast, PPN find that for fractonic RQCs $S_{\mathrm{E}}(t)$ plateaus at a value independent of the partition size, consistent with a failure to thermalize.

PPN's work establishes a new mechanism of ergodicity breaking - one which might possibly extend to three-dimensional systems. Memory of initial conditions is preserved by the dynamics, even in the presence of noise. A tantalizing possibility suggested by their analysis is that one and two-dimensional translationally-invariant fracton systems subject to Hamiltonian dynamics should exhibit robust, bona fide (i.e. no asymptotic delocalization) MBL, since PPN's arguments do not invoke the analytical apparatus (i.e. locator expansions) upon which MBL no-go theorems rest.

There are two significant caveats. First, the numerical results obtained are for modest system sizes (up to $L=21$ sites). Second, and more crucially, is that all the above results 
apply only to initial conditions in which there is a vanishing fracton charge density, and are in that sense finely tuned. For generic initial states, where the typical spacing $l$ between fracton charges is finite, dipoles may be emitted at one location and absorbed at another. This implies a finite diffusion constant $D \sim l^{-2}$ for the fracton charges themselves. PPN find that this gives rise to a coarsening behavior in which fracton charges asymptotically coalesce at their center of mass at long times. The dynamics of this coarsening, and its consequences for entanglement growth and saturation, are as yet unknown.

\section{References}

[1] C. W. von Keyserlingk, T. Rakovszky, F. Pollmann, and S. L. Sondhi, Phys. Rev. X, 8, 021013 (2018); A. Nahum, S. Vijay, and J. Haah, Phys. Rev. X, 8, 021014 (2018).

[2] T. Rakovszky, F. Pollmann, and C. W. von Keyserlingk, arXiv:1710.09827; V. Khemani, A. Vishwanath, and D. Huse, arXiv:1710.09835.

[3] C. Chamon, Phys. Rev. Lett. 94, 040402 (2005); R. M. Nandkishore and M. Hermele, arXiv:1803.11196.

[4] A. Prem, J. Haah, and R. Nandkishore, Phys. Rev. B, 95, 155133 (2017). 